\title{
Beam misalignments and fluid velocities in laser-induced thermal acoustics
}

\author{
Stefan Schlamp, Eric B. Cummings, and Hans G. Hornung
}

\begin{abstract}
Beam misalignments and bulk fluid velocities can influence the time history and intensity of laserinduced thermal acoustics (LITA) signals. A closed-form analytic expression for LITA signals incorporating these effects is derived, allowing the magnitude of beam misalignment and velocity to be inferred from the signal shape. It is demonstrated how instantaneous, nonintrusive, and remote measurement of sound speed and velocity (Mach number) can be inferred simultaneously from homodyne-detected LITA signals. The effects of different forms of beam misalignment are explored experimentally and compared with theory, with good agreement, allowing the amount of misalignment to be measured from the LITA signal. This capability could be used to correct experimental misalignments and account for the effects of misalignment in other LITA measurements. It is shown that small beam misalignments have no influence on the accuracy or repeatability of sound speed measurements with LITA. (C) 1999 Optical Society of America
\end{abstract}

OCIS codes: $\quad 000.2170,050.2770,120.6780,120.7280,190.4380$.

\section{Introduction}

The four-wave mixing technique of laser-induced thermal acoustics (LITA) is used in various laboratories to measure sound speeds and thermal diffusivity $^{1-3}$ as well as fluid velocities. ${ }^{4}$ A gratingshaped pressure and temperature perturbation is created in a fluid by two coherent intersecting pulsed laser beams. These perturbations evolve hydrodynamically in time and space. This evolution can be examined when a third continuous laser beam is focused on the associated density gratings at its Bragg angle. Depending on the modulation depth of the density grating, a fraction of this source beam is coherently scattered into a weak signal beam. The induced acoustic waves move outward, modulating the density field and hence the signal at the grating Brillouin frequency, or the frequency of sound waves with the grating wavelength. Hence one approach to measure the sound speed is to measure the frequency of the signal modulation by means of a fre-

S. Schlamp and H. G. Hornung are with the Graduate Aeronautical Laboratories, California Institute of Technology, Pasadena, California 91125. E. B. Cummings is with Sandia National Laboratories, Livermore, California 94551. The e-mail address for S. Schlamp is stefan@galcit.caltech.edu.

Received 1 April 1999; revised manuscript received 21 June 1999.

0003-6935/99/275724-10\$15.00/0

(C) 1999 Optical Society of America quency decomposition method. However, finite signal lifetime and sampling resolution limits the accuracy of this technique.

Another approach is to model the physics of the interactions that produce the LITA signal and derive an analytic expression for the signal as a function of experimental parameters, fluid properties including sound speeds and thermal diffusivity, and fluid velocity. A least-squares fit of this expression to experimental signals then provides best estimates of the signal parameters and hence fluid properties. Cummings et al..$^{5}$ derived such an expression that includes thermalization and electrostriction as the two main mechanisms for the creation of the grating. However, the assumption was made that the system was in perfect optical alignment and that the fluid was at rest. Obviously this approach breaks down when these assumptions are grossly violated. In these cases, the theory is not a valid representation of the experiment, and the fitting procedure returns erroneous values.

The research presented here extends the earlier theory to include the effects of a convection velocity and finite beam misalignment. These may then be measured through the least-squares fitting procedure, simplifying LITA velocimetry. Present velocimetry techniques that include LITA rely on the measurement of a small Doppler shift of the signal beam by use of heterodyne detection. Now, from only the recorded signal and by use of the simpler homodyne detection, sound speeds, velocities, and 


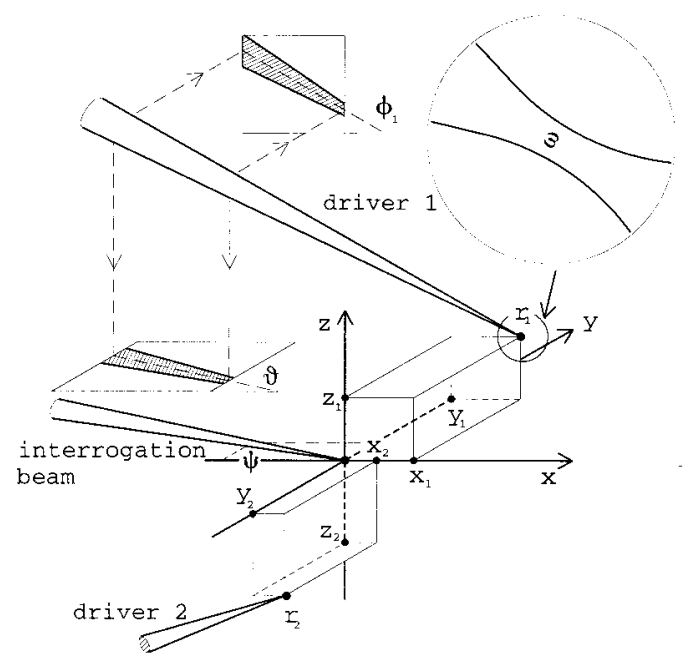

Fig. 1. Frame of reference for LITA analysis. The beams' diameters at their foci are $\omega$ and $\sigma$ for the driver beams and interrogation beam, respectively.

transport properties of the fluid can be extracted simultaneously. In addition we can extract beam misalignment measurements from the signal and either realign the optics with this information or take them into account in the data analysis.

In Sections 2-6 we extend the LITA analysis of Ref. 5 using nomenclature adapted mostly from that derivation. First, the grating creation that is due to the optoacoustic forcing is modeled, and the functional form of the electric field grating in the sample volume is derived. Next, the fluid response to the electric field is examined. Because the underlying physics have not changed, the fluid response to electric fields has been left unchanged from Ref. 5; only the results are given, and the interested reader is referred to Refs. 5 and 6 . Finally, we model the scattering of the source beam into the signal beam using the linearized equation of light scattering.

In Sections 7-9 we present the experimental results that validate the theory.

\section{Frame of Reference}

We define our frame of reference so that the origin of our fixed Cartesian coordinate system is at the focus of the source beam as shown in Fig. 1. The source beam lies in the $x-y$ plane and forms an angle $\psi$ with the $x$ axis. The $x-z$ plane bisects the two driver beams so that each one intersects this plane at an angle $\theta$. They intersect the $x-y$ plane at angles $\phi_{1}$ and $\phi_{2}$ (angular misalignment). Their foci are at $\mathbf{r}_{1}$ and $\mathbf{r}_{2}$, respectively (spatial misalignment). We denote the time of the short driver pulse as $t=0$. A perfectly aligned LITA setup would have $\mathbf{r}_{1}=\mathbf{r}_{2}=$ $\phi_{1}=\phi_{2}=0$ but not $\theta=0$.

\section{Optoacoustic Forcing}

We first model the electric field grating in the sample volume defined by the shallow-angle intersection of the two driver beams. We assume that each driver laser beam has a Gaussian profile with Gaussian half-width $\omega$ and denote the normalized electric fields of the two driver lasers by $\mathbf{E}_{d 1}(\mathbf{r}, t)$ and $\mathbf{E}_{d 2}(\mathbf{r}, t)$, respectively, so that

$$
\mathbf{E}_{d 1}=\mathbf{E}_{1}+\mathbf{E}_{1}^{*}, \quad \mathbf{E}_{d 2}=\mathbf{E}_{2}+\mathbf{E}_{2}{ }^{*}
$$

where ()$^{*}$ denotes the complex conjugate. Then

$$
\begin{aligned}
\mathbf{E}_{1}= & \frac{\mathscr{E}(t)}{2} \sqrt{\frac{2}{\pi \omega^{2}}} \exp \left[i k_{d} \hat{e}_{1} \cdot\left(\mathbf{r}-\mathbf{r}_{1}\right)-i f_{d} t\right] \\
& \times \exp \left[-\left|\frac{\hat{e}_{1} \otimes\left(\mathbf{r}-\mathbf{r}_{1}\right)}{\omega}\right|^{2}\right], \\
\mathbf{E}_{2}= & \frac{\mathscr{E}(t)}{2} \sqrt{\frac{2}{\pi \omega^{2}}} \exp \left[i k_{d} \hat{e}_{2} \cdot\left(\mathbf{r}-\mathbf{r}_{2}\right)-i f_{d} t\right] \\
& \times \exp \left[-\left|\frac{\hat{e}_{2} \otimes\left(\mathbf{r}-\mathbf{r}_{2}\right)}{\omega}\right|^{2}\right],
\end{aligned}
$$

where $\mathscr{E}(t)$ is the temporal electric field envelope, $\otimes$ denotes the vector cross product, $f_{d}$ is the driver laser frequency, $k_{d}$ is the wave-vector magnitude, $\mathbf{r}_{1}=\left(x_{1}\right.$, $\left.y_{1}, z_{1}\right)$ and $\mathbf{r}_{2}=\left(x_{2}, y_{2}, z_{2}\right)$ are the Cartesian coordinates of the foci of the two beams, respectively, and

$$
\begin{aligned}
& \hat{e}_{1}=\left(\cos \theta \cos \phi_{1}, \sin \theta, \sin \phi_{1}\right), \\
& \hat{e}_{2}=\left(\cos \theta \cos \phi_{2},-\sin \theta, \sin \phi_{2}\right)
\end{aligned}
$$

are unit vectors in the direction of the driver beams, where $\theta$ is the driver beam crossing half-angle and $\phi_{1}$ and $\phi_{2}$ are the angular misalignments of the driver beams. One assumption that is made is that the driver beams are of equal strength and geometry. This will be impossible to achieve experimentally. Deviations from this condition result in an otherwise equivalent grating with a Gaussian background intensity ${ }^{6-8}$ that will cause no significant scattering of the source beam at the grating Bragg angle, so this assumption can safely be made. To keep the algebra as compact as possible, angular misalignments are dropped at this point, i.e., $\phi_{1}=\phi_{2}=0$. Also note that, because the driver pulse is short compared with all the other relevant time scales, we assume that the fluid does not move during the pulse and so any velocity terms [not shown in Eqs. (1b) and (1c)] can be safely neglected for the optoacoustic forcing process. They have to be included, however, for the grating evolution which takes several orders of magnitude longer. Now we calculate the electric field grating intensity to be

$$
\begin{aligned}
I & =\left|\mathbf{E}_{d 1}+\mathbf{E}_{d 2}\right|^{2}=\left(\mathbf{E}_{d 1}+\mathbf{E}_{d 2}\right)\left(\mathbf{E}_{d 1}+\mathbf{E}_{d 2}\right)^{*} \\
& \cong 2\left(\mathbf{E}_{1} * \mathbf{E}_{2}+\mathbf{E}_{1} \mathbf{E}_{2}^{*}\right)=E_{d} I_{d} P_{d}(t),
\end{aligned}
$$

where only the cross terms are retained in the first step as the other terms do not add spatially oscillating contributions but only a slowly spatially varying background, similar to the case of the not equally strong driver beams mentioned above. In the second step we decompose the intensity into the total driver laser energy $E_{d}$, the normalized spatial grating intensity distribution $I_{d}$, and the normalized driver laser temporal 
profile $P_{d}$ which we approximate by a Dirac delta function at a later point. The result for $I_{d}$ is

$$
\begin{aligned}
I_{d}= & \frac{2}{\pi \omega^{2}} \cos \left\{2 k_{d}[-\xi \cos \theta+(y-\bar{\eta}) \sin \theta]\right\} \\
& \times \exp \left\{-\frac{2}{X^{2}}\left[x-\left(\bar{\xi}-\frac{X}{Y} \eta\right)\right]^{2}\right. \\
& \left.-\frac{2}{Y^{2}}\left[y-\left(\bar{\eta}-\frac{Y}{X} \xi\right)\right]^{2}-\frac{2}{Z^{2}}\left[(z-\bar{\zeta})^{2}+\xi^{2}\right]\right\},
\end{aligned}
$$

where

$$
\begin{array}{rlrl}
X & =\frac{\omega}{\sin \theta}, & Y=\frac{\omega}{\cos \theta}, & Z=\omega, \\
\bar{\xi}=\left(x_{1}+x_{2}\right) / 2, & \bar{\eta}=\left(y_{1}+y_{2}\right) / 2, & \bar{\zeta}=\left(z_{1}+z_{2}\right) / 2, \\
\xi=\left(x_{1}-x_{2}\right) / 2, & \eta=\left(y_{1}-y_{2}\right) / 2, & \zeta=\left(z_{1}-z_{2}\right) / 2 .
\end{array}
$$

At this point we use the fact that the beam crossing angle is small and that hence $X \gg Y, Z$. Consequently we neglect all variations in the $x$ direction including the misalignments. The side effect of this assumption together with the assumption of no angular misalignments is that we exclude any rotation of the grating, i.e., it is always perpendicular to the $y$ direction. The proper limit of Eq. (3a) is then

$$
\begin{aligned}
I_{d}= & \frac{2}{\pi \omega^{2}} \exp \left\{-\frac{2}{Y^{2}}\left[(y-\bar{\eta})^{2}+\eta^{2}\right]\right. \\
& \left.-\frac{2}{Z^{2}}\left[(z-\bar{\zeta})^{2}+\zeta^{2}\right]\right\} \cos \left[q_{\psi}(y-\bar{\eta})\right],
\end{aligned}
$$

with $q_{\psi}=2 k_{d} \sin \theta$. Equation (4) represents a fringe pattern perpendicular to the $y$ direction with a Gaussian intensity profile centered at $(0, \bar{\eta}, \bar{\zeta})$.

At a later point we will have to take the threedimensional convolution of $I_{d}$ with Green's function of the fluid response to the electric field. We perform this operation by multiplying the expressions in Fourier space. The spatial Fourier transform of Eq. (4) is

$$
\begin{aligned}
I_{d}(\mathbf{q})= & \frac{Y Z}{2 \omega^{2}} \exp \left(-\frac{2}{Y^{2}} \eta^{2}-\frac{2}{Z^{2}} \zeta^{2}-\frac{Z^{2}}{8} q_{z}^{2}\right. \\
& \left.+i \bar{\eta} q_{y}+i \bar{\zeta} q_{z}\right) \times\left\{\exp \left[-\frac{Y^{2}}{8}\left(q_{y}-q_{\psi}\right)^{2}\right]\right. \\
& \left.+\exp \left[-\frac{Y^{2}}{8}\left(q_{y}+q_{\psi}\right)^{2}\right]\right\} .
\end{aligned}
$$

\section{Grating Evolution}

Here one invokes an analysis of optoacoustic effects and evolution of the resulting hydrodynamic disturbances to model how the fluid responds to the electric field grating.

Two such effects have been observed in LITA. Thermalization is the process of absorption of driver laser energy by the fluid. The fluid temperature in- creases locally where the electric field grating intensity is high. The temperature perturbations are typically fractions of $1 \mathrm{~K}$. This heating process that is due to superelastic collisions typically occurs quickly compared to the Brillouin period of the grating. In the short duration of the driver pulse, no bulk fluid displacement can occur. Therefore the density field cannot change during this time frame. Linear hydrodynamics show that this field may be decomposed into the superposition of three density perturbation gratings that cancel at $t=0$. One grating is due to the temperature grating and represents the equilibrium solution. It is offset by two density gratings of opposite sign and half the amplitude. For $t>0$ these propagate in opposite directions at the local speed of sound. The second effect is electrostriction, which describes the effect that polarizable molecules accelerate along or against an electric field gradient. As with thermalization, the driver pulse is too short for bulk fluid displacement. As a result, two acoustic waves of opposite sign are created at $t=0$. For $t>0$, they propagate in opposite directions. If we briefly assume our frame of reference to move with the fluid, we can adopt the analysis of the optoacoustic response of the fluid to the electric field from Ref. 5 and, to switch back to our original fixed coordinate system, superimpose a (constant) external fluid velocity $\mathbf{u}=(0, v, w)$.

Starting from the linearized equations of motion with forcing terms for electrostriction (in the momentum equation) and for thermalization (in the energy equation), Green's functions $H_{\theta}$ and $H_{e}$ for the optoacoustic response of the fluid to thermalization and electrostriction, respectively, are

$$
\begin{aligned}
& H_{\theta}(q, t)=H_{\theta P_{1}} \Phi_{P_{1}}+H_{\theta P_{2}} \Phi_{P_{2}}+H_{\theta T} \Phi_{T}+H_{\theta D} \Phi_{D}, \\
& H_{e}(q, t)=H_{e P_{1}} \Phi_{P_{1}}+H_{e P_{2}} \Phi_{P_{2}}+H_{e T} \Phi_{T},
\end{aligned}
$$

where

$$
\begin{aligned}
\Phi_{P_{1,2}}(q, t) & =\exp \left(-\Gamma q^{2} t \pm i c_{s} q t+i v t q_{y}+i w t q_{z}\right), \\
\Phi_{T}(q, t) & =\exp \left(-D_{T} q^{2} t+i v t q_{y}+i w t q_{z}\right), \\
\Phi_{D}(q, t) & =\exp \left[-\left(\gamma_{\theta}+\gamma_{n \theta}\right) t-D_{s} q^{2} t+i v t q_{y}+i w t q_{z}\right]
\end{aligned}
$$

$$
\begin{aligned}
H_{\theta P_{1}} & =\frac{[1+i(\Delta-G)](1-G \Pi-i \Pi)}{2\left[1+(\Delta-G)^{2}\right]\left[(1-G \Pi)^{2}+\Pi^{2}\right]}=H_{\theta P_{2}}{ }^{*}, \\
H_{\theta_{T}} & =-\frac{1}{\left[1+(\Delta-G)^{2}\right](1-\Delta \Pi)}, \\
H_{\theta_{D}} & =\frac{\Pi^{2}}{\left[(1-G \Pi)^{2}+\Pi^{2}\right](1-\Delta \Pi)}, \\
H_{e P_{1}} & =i \frac{[1-i(\gamma \Delta-G)][1+i(\Delta-G)]}{2\left[1+(\Delta-G)^{2}\right]}=H_{e P_{2}}{ }^{*}, \\
H_{e T} & =\frac{(\gamma-1) \Delta}{\left[1+(\Delta-G)^{2}\right]}, \\
\Pi & =c_{s} q /\left(\gamma_{\theta}+\gamma_{n \theta}+D_{s} q^{2}\right),
\end{aligned}
$$


$\Delta$ is the thermal grating damping over a period of acoustic motion, $\Gamma$ is the acoustic damping rate $\{\Gamma=$ $\left.1 / 2\left[(\gamma-1) D_{T}+D_{V}\right]\right\}, \Pi$ is the ratio of the soundwave frequency with the wave vector $q_{y}$ to the excited-state energy decay rate, $\gamma$ is the ratio of specific heats, $D_{T}$ is the thermal diffusivity, $D_{V}$ is the longitudinal kinematic diffusivity of the fluid, $D_{S}$ is the diffusivity of excited-state target molecules, $\gamma_{\theta}$ is the rate of excited-state energy decay caused by thermalization, and $\gamma_{n \theta}$ is the energy decay rate that is not due to thermalization.

$\Phi_{P_{1}}$ and $\Phi_{P_{2}}$ are associated with the acoustic waves traveling in opposite directions, $\Phi_{T}$ is related to the density perturbations caused by the thermal grating, and $\Phi_{D}$ is due to the finite thermalization rate. A single-rate thermalization process is assumed. The density-time behavior is then

$$
\frac{\rho^{\prime}(\mathbf{q}, t)}{\rho}=-\omega^{2} I_{d} P_{d}(t) \circ\left(H_{\theta} U_{\theta}+H_{e} U_{e}\right),
$$

where $U_{\theta}$ and $U_{e}$ are the approximate modulation depths of the thermalization and the electrostriction grating, respectively, and $\circ$ denotes a temporal convolution.

$I_{d}(\mathbf{q})$ is peaked at $\mathbf{q}=\left(0, q_{\psi}, 0\right)$. Therefore, approximate to first order, $q \approx q_{y}$. This implies the assumption of plane-wave fronts which is a good approximation if the waves have not traveled too far from their point of origin and if the fringe spacing is small. Also note that $q_{z}=0$ implies a constant intensity in the $q_{y}$ direction when in fact the intensity has a Gaussian profile in this direction. But the error is only a multiplicative factor because all grating cross sections perpendicular to $q_{z}$ are self-similar.

Taking the inverse Fourier transform of the products

$$
\Phi_{\left(P_{1}, P_{2}, T, D\right)}^{(d)}=I_{d}(\mathbf{q}) \Phi_{\left(P_{1}, P_{2}, T, D\right)}(\mathbf{q}, t)
$$

we obtain

$$
\begin{aligned}
\Phi_{P_{1,2}}^{(d)}(\mathbf{r}, t)= & \frac{2}{\pi \omega^{2}} \exp \left(-\frac{2}{Y^{2}} \eta^{2}-\frac{2}{Z^{2}} \zeta^{2}\right) \exp \left(-\Gamma q_{\psi}{ }^{2} t\right) \\
& \times \cos \left\{\left[y-\left(v \pm c_{s}\right) t\right] q_{\psi}\right\} \\
& \times \exp \left(-\frac{2}{Y^{2}}\left\{y-\left[\bar{\eta}+\left(v \pm c_{s}\right) t\right]\right\}^{2}\right. \\
& \left.-\frac{2}{Z^{2}}[z-(\bar{\zeta}+w t)]^{2}\right) \\
\Phi_{T}^{(d)}(\mathbf{r}, t)= & \frac{2}{\pi \omega^{2}} \exp \left(-\frac{2}{Y^{2}} \eta^{2}-\frac{2}{Z^{2}} \zeta^{2}\right) \exp \left(-D_{T} q_{\psi}{ }^{2} t\right) \\
& \times \cos \left[(y-v t) q_{\psi}\right] \\
& \times \exp \left\{-\frac{2}{Y^{2}}[y-(\bar{\eta}+v t)]^{2}\right. \\
& \left.-\frac{2}{Z^{2}}[z-(\bar{\zeta}+w t)]^{2}\right\}
\end{aligned}
$$

$$
\begin{aligned}
\Phi_{D}^{(d)}(\mathbf{r}, t)= & \frac{2}{\pi \omega^{2}} \exp \left(-\frac{2}{Y^{2}} \eta^{2}-\frac{2}{Z^{2}} \zeta^{2}\right) \exp \left[-D_{S} q_{\psi}{ }^{2} t\right. \\
& \left.-\left(\gamma_{\theta}+\gamma_{n \theta}\right) t\right] \cos \left[(y-v t) q_{\psi}\right] \\
& \times \exp \left\{-\frac{2}{Y^{2}}[y-(\bar{\eta}+v t)]^{2}\right. \\
& \left.-\frac{2}{Z^{2}}[z-(\bar{\zeta}+w t)]^{2}\right\} .
\end{aligned}
$$

In Eqs. (9a)-(9c) the assumption is made that the length scales do not change because of diffusion over the LITA time scales. Also, a phase shift of $\bar{\eta} q_{\psi}$ was dropped in all three cosine terms.

\section{Acousto-Optical Scattering}

After describing the creation and time evolution of the density grating in the sample volume, our next step is to model how a continuous-source beam focused at the origin and incident on the grating at the phase-matched angle $\psi$ scatters off the grating into the coherent signal beam.

If we assume the density variations $\rho^{\prime}$ to be small, we can use the linearized equation of light scattering. ${ }^{9}$ A narrow-band source beam $\mathbf{E}_{0}(\mathbf{r}, t) \cos \left(f_{0} t\right)$ scatters into the electric field $\mathbf{E}_{s}$ by a small disturbance in the susceptibility $\chi(\mathbf{r}, t ; f)$. In the far field,

$$
\mathbf{E}_{s}(\mathbf{R}, t ; \mathbf{q})=-\frac{k_{s}{ }^{2}}{4 \pi R} \cos \left(\mathbf{k}_{s} \cdot \mathbf{R}-f_{0} t\right) \mu(\mathbf{q}, t),
$$

where $k_{s}$ is the wave vector of the scattered beam, $f_{0}$ is the frequency of the source beam, $\mathbf{R}$ is the position vector relative to the scatterer, and $\mu(\mathbf{q}, t)$ is the Fourier transform of the overlap of the susceptibility grating and the source laser field:

$$
\mu(\mathbf{r}, t)=\chi^{\prime}\left(\mathbf{r}, t ; f_{0}\right) \mathbf{E}_{0}(\mathbf{r}, t) .
$$

Just as with the driver beams, we assume the source beam to have a spatial Gaussian intensity profile. Hence

$$
\begin{aligned}
\mathbf{E}_{S}= & \mathbf{E}_{0}+\mathbf{E}_{0}{ }^{*}, \\
\mathbf{E}_{0}= & \frac{1}{2} \exp \left(i k_{0} \hat{e}_{0} \cdot \mathbf{r}\right. \\
& \left.-i f_{0} t\right) \exp \left(-\left|\frac{\hat{e}_{0} \otimes \mathbf{r}}{\sigma}\right|^{2}\right), \\
\hat{e}_{0}= & (\cos \psi, \sin \psi, 0) .
\end{aligned}
$$

Proceeding as before with the driver lasers, the electric field of the source laser is $\mathbf{E}_{S}(\mathbf{r}, t)=$ $P_{0}(t) I_{0}(\mathbf{r}) \cos \left(f_{0} t\right)$ with

$$
I_{0}=\sqrt{\frac{2}{\pi \sigma^{2}}} \exp \left[-\left(\frac{y}{\sigma_{y}}\right)^{2}-\left(\frac{z}{\sigma_{z}}\right)^{2}\right],
$$


where $\sigma$ is the Gaussian half-width of the source laser and

$$
\sigma_{y}=\frac{\sigma}{\sin \psi}, \quad \sigma_{z}=\sigma
$$

In Eqs. (13a) and (13b) we neglect variations in the $x$ direction by assuming $\sigma_{x}=\sigma / \sin \psi \gg \sigma_{y}, \sigma_{z}$. We define $\Phi_{\left(P_{1},{ }_{P},{ }_{P_{2}}, T, D\right)}^{(d, \mathbf{r}, t)}=I_{0}(\mathbf{r}) \Phi_{\left(P_{1}, P_{2}, T, D\right)}^{(d)}$, the field of the overlap of the source beam and the evolving density grating. Taking the Fourier transform of these products, we can write

$$
\Phi_{\left(P_{1}, P_{2}, T, T\right)}^{(d, 0)}(\mathbf{q}, t)=N \Psi_{\left(P_{1}, P_{2}, T, D\right)}(\mathbf{q}, t) \sum_{\left(P_{1}, P_{2}, T, D\right)}(t),
$$

where

$$
\begin{aligned}
& N=\frac{\sqrt{2 / \pi}}{\sigma \omega^{2}}\left(\frac{Y^{2} \sigma_{y}^{2}}{Y^{2}+2 \sigma_{y}^{2}}\right)^{1 / 2}\left(\frac{Z^{2} \sigma_{z}^{2}}{Z^{2}+2 \sigma_{z}^{2}}\right)^{1 / 2} \\
& \times \exp \left(-\frac{2}{Y^{2}} \eta^{2}-\frac{2}{Z^{2}} \zeta^{2}\right) \text {, } \\
& \Psi_{P_{1,2}}=\exp \left\{-\frac{1}{4} \frac{Y^{2} \sigma_{y}{ }^{2}}{Y^{2}+2 \sigma_{y}{ }^{2}}\left(q_{y}-q_{\psi}\right)^{2}\right. \\
& \left.+i \frac{2 \sigma_{y}{ }^{2}}{Y^{2}+2 \sigma_{y}{ }^{2}}\left[\bar{\eta}+\left(v \pm c_{s}\right) t\right]\left(q_{y}-q_{\psi}\right)\right\} \\
& \times \exp \left[-\frac{1}{4} \frac{Z^{2}{\sigma_{z}}^{2}}{Z^{2}+2 \sigma_{z}^{2}} q_{z}^{2}\right. \\
& \left.+i \frac{2 \sigma_{z}^{2}}{Z^{2}+2 \sigma_{z}^{2}}(\bar{\zeta}+w t) q_{z}\right], \\
& \Psi_{T}=\exp \left[-\frac{1}{4} \frac{Y^{2} \sigma_{y}{ }^{2}}{Y^{2}+2 \sigma_{y}{ }^{2}}\left(q_{y}-q_{\psi}\right)^{2}\right. \\
& \left.+i \frac{2 \sigma_{y}^{2}}{Y^{2}+2 \sigma_{y}^{2}}(\bar{\eta}+v t)\left(q_{y}-q_{\psi}\right)\right] \\
& \times \exp \left[-\frac{1}{4} \frac{Z^{2}{\sigma_{z}}^{2}}{Z^{2}+2 \sigma_{z}^{2}} q_{z}^{2}\right. \\
& \left.+i \frac{2 \sigma_{z}^{2}}{Z^{2}+2 \sigma_{z}^{2}}(\bar{\zeta}+w t) q_{z}\right] \\
& =\Psi_{D} \text {, } \\
& \Sigma_{P_{1,2}}=\exp \left\{-\Gamma q_{\psi}{ }^{2} t-\frac{2\left[\bar{\eta}+\left(v \pm c_{s}\right) t\right]^{2}}{Y^{2}+2 \sigma_{y}{ }^{2}}\right. \\
& \left.-\frac{2}{Z^{2}+2 \sigma_{z}^{2}}(\bar{\zeta}+w t)^{2}+i q_{\psi}\left(v \pm c_{s}\right) t\right\}, \\
& \Sigma_{T}=\exp \left[-D_{T} q_{\psi}{ }^{2} t-\frac{2(\bar{\eta}+v t)^{2}}{Y^{2}+2 \sigma_{y}{ }^{2}}-\frac{2(\bar{\zeta}+w t)^{2}}{Z^{2}+2 \sigma_{z}{ }^{2}}\right. \\
& \left.+i q_{\psi} v t\right] \text {, } \\
& \Sigma_{D}=\exp \left[-D_{s} q_{\psi}{ }^{2} t-\left(\gamma_{\theta}+\gamma_{n \theta}\right) t-\frac{2(\bar{\eta}+v t)^{2}}{Y^{2}+2 \sigma_{y}{ }^{2}}\right. \\
& \left.-\frac{2(\bar{\zeta}+w t)^{2}}{Z^{2}+2 \sigma_{z}^{2}}+i q_{\psi} v t\right] \text {. }
\end{aligned}
$$

In Eqs. (14c) the contributions from a similar lobe centered at $q_{y}=-q_{\psi}$ are neglected. Using this result in Eq. (10) yields the electric field of the signal beam:

$$
\begin{aligned}
\frac{\mathbf{E}_{s}(\mathbf{q}, \mathbf{R}, t)}{P_{0}(t)}= & -\frac{k_{s}{ }^{2} \omega^{2}}{4 \pi R} \chi\left(f_{0}\right) \exp \left[i \left(\mathbf{k}_{s} \cdot \mathbf{R}\right.\right. \\
& \left.\left.-f_{0} t\right)\right] P_{d}(t) \circ \mathscr{R}\left[A_{P_{1}} \Phi_{P_{1}}^{(d, 0)}+A_{P_{2}} \Phi_{P_{2}}^{(d, 0)}\right. \\
& \left.+A_{T} \Phi_{T}^{(d, 0)}+A_{D} \Phi_{D}^{(d, 0)}\right],
\end{aligned}
$$

where we used

$$
\begin{aligned}
A_{P_{1,2}} & =U_{\theta} H_{\theta P_{1,2}}+U_{e} H_{e P_{1,2}} \\
A_{T} & =U_{\theta} H_{\theta T}+U_{e} H_{e T}, \quad A_{D}=U_{\theta} H_{\theta D},
\end{aligned}
$$

and where $\mathscr{R}($ ) denotes the real part.

\section{Detected Laser-Induced Thermal Acoustics Signal}

The detected LITA signal by use of heterodyne detection is then simply the integral of Eqs. (15) over the detection angle where the detector is centered at $\mathbf{q}=$ $\left(0, q_{\psi}, 0\right)$. In the limit for a small detector, Eqs. (15) multiplied by the detection angle yield the LITA signal. In the experiments presented here, homodyne detection is used. The detector measures the intensity of the electric field, i.e., the square of the modulus of Eqs. (15):

$$
\begin{aligned}
\frac{\mathscr{L}_{\mathrm{hom}}}{P_{0}^{2}(t)}= & \frac{k_{0} \omega^{4}}{16 \pi^{2} \cos ^{2} \psi}\left|\chi\left(f_{0}\right)\right|^{2} \\
& \times\left[A_{P_{1}} \Phi_{P_{1}}^{(d, 0)}+A_{P_{1}} \Phi_{P_{2}}^{(d, 0)}+A_{T} \Phi_{T}^{(d, 0)}+A_{D} \Phi_{D}^{(d, 0)}\right] \\
& \times\left[A_{P_{1}}{ }^{*} \Phi_{P_{1}}^{(d, 0)} * A_{P_{1}}{ }^{*} \Phi_{P_{2}}^{(d, 0) *}+A_{T} \Phi_{T}^{(d, 0) *}\right. \\
& \left.+A_{D} \Phi_{D}^{(d, 0) *}\right] .
\end{aligned}
$$

In Eq. (16) we used the fact that the driver pulse $(\approx 7$ $\mathrm{ns}$ ) is short compared to the inverse Brillouin frequency, and it was approximated by a Dirac delta function that eliminated the double temporal convolution over the driver pulse time history.

In the large detector limit the integration over the detection angle can be approximated by infinite integrals. The final result is then

$$
\begin{aligned}
\frac{\mathscr{L}_{\mathrm{hom}}}{P_{0}^{2}(t)}= & \frac{k_{0}{ }^{2}}{4 \pi^{2} \cos ^{2} \psi}\left|\chi\left(f_{0}\right)\right|^{2} \exp \left(-\frac{2}{Y^{2}} \eta^{2}-\frac{2}{Z^{2}} \zeta^{2}\right) \\
& \times\left\{\exp \left[-\frac{8 \sigma_{y}{ }^{2}}{Y^{2}\left(Y^{2}+2 \sigma_{y}^{2}\right)}\left(\frac{c_{s} t}{2}\right)^{2}\right]\right. \\
& \times\left[\left(P_{1}+P_{2}\right)\left(T^{*}+D^{*}\right)+\left(P_{1}^{*}+P_{2}{ }^{*}\right)(T+D)\right] \\
& +\exp \left[-\frac{8 \sigma_{y}{ }^{2}}{Y^{2}\left(Y^{2}+2 \sigma_{y}^{2}\right)}\left(c_{s} t\right)^{2}\right]\left(P_{1} P_{2}{ }^{*}+P_{1}{ }^{*} P_{2}\right) \\
& +\left(P_{1} P_{1} *+P_{2} P_{2}{ }^{*}+T T^{*}+T D^{*}+T^{*} D\right. \\
& \left.\left.+D D^{*}\right)\right\}
\end{aligned}
$$




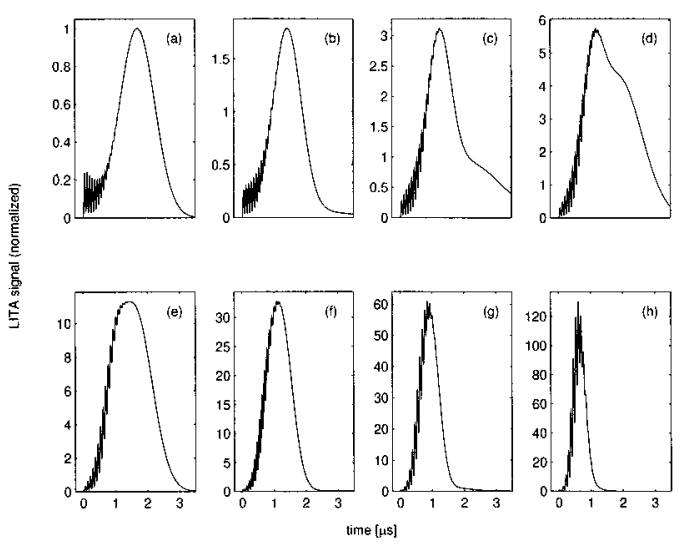

Fig. 2. Theoretical LITA signals for atmospheric air from Eq. (17) with $\theta=1.23^{\circ}, \omega=370 \mu \mathrm{m}, \sigma=700 \mu \mathrm{m}$, misalignment $\bar{\eta}=-2 \omega$, and with a fluid flow in $+y$ direction with different Mach numbers: (a) $M=0$, (b) $M=0.25$, (c) $M=0.5$, (d) $M=0.75$, (e) $M=1.0$, (f) $M=1.5$, (g) $M=2.0$, and (h) $M=3.0$.

where $P_{1}=A_{P_{1}} \Sigma_{P_{1}}, T^{*}=A_{T} * \Sigma_{T}^{*}$, etc., and where the symbols $A_{\left(P_{1}, P_{2}, T, D\right)}$ and $\Sigma_{\left(P_{1}, P_{2}, T, D\right)}$ are defined in Eqs. $(15 \mathrm{~b})$ and $(14 \mathrm{~d})$, respectively.

In the absence of beam misalignments and fluid velocities, Eq. (17) collapses to the solution in Ref. 5 with the exception of a multiplicative constant. This difference is due to the fact that variations in the $x$ direction were dropped much earlier than in Ref. 5 .

In the absence of bulk fluid velocities, only the misalignment $\bar{\eta}$ changes the time history of the LITA signal whereas $\eta, \zeta$, and $\bar{\zeta}$ simply decrease the signal intensity. Also, Eq. (17) is symmetric with respect to all possible misalignments. In the absence of beam misalignments, both fluid velocities have a similar influence on the signal that resembles additional acoustic damping and thermal diffusion. Among all misalignment and velocity components, $\bar{\eta}$ and $v$ are the most interesting. In particular, by introducing a known $\bar{\eta}$ it is possible for one to measure the time it takes the density grating to travel to the interrogation beam, thereby obtaining the velocity component $v$ more precisely than by means of an enhanced decay rate. Figure 2 shows how intentionally misaligning the beam can produce a dependence of the signal on velocity that is more orthogonal to other parameters and therefore more accurately inferred from signals. Note that oscillations are still present in the signal so that the sound speed can also be measured. Depending on the range of velocities to be measured, suitable values for $\bar{\eta}, \omega$, and $\theta$ can be chosen.

\section{Experimental Setup}

Figure 3 depicts a schematic diagram of the experimental setup. A $Q$-switched, frequency-doubled $\mathrm{Nd}$ : YAG laser (Spectra-Physics GCR-150-10) drives a dye laser emitting $\sim 10-\mathrm{mJ}, 7-\mathrm{ns}$ pulses at $589 \mathrm{~nm}$ with a repetition rate of $10 \mathrm{~Hz}$. Behind iris $i 2$, the beam splitter (bs) splits the beam into approximately equal-intensity halves. The beam splitter and mirrors $m 3, m 4$, and $m 5$ are placed to match the path

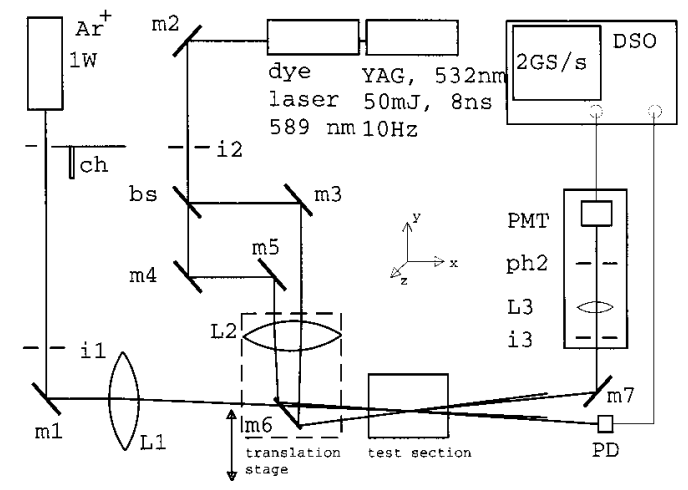

Fig. 3. Schematic diagram of experimental LITA setup.

length of the two beams to within $\sim 1 \mathrm{~mm}$. Lens $l 2$ with a focal length of $750 \mathrm{~mm}$ focuses both beams onto the sample volume in the test section. Lens $l 2$ and mirror $m 6$ are mounted on a translation stage with a $10-\mu \mathrm{m}$-resolution micrometer drive. The test section is a high-pressure bomb with optical access through antireflection-coated BK-7 windows on opposite sides.

A cw argon-ion laser (Spectra Physics, Model 165) at $488 \mathrm{~nm}$ provides $0.5 \mathrm{~W}$ for the source beam. A chopper wheel (ch) (Scitech Instruments, Optical Chopper) blocks the beam except for the 1-ms duration pulses that are synchronized (LabSmith LC880) with the $Q$-switch trigger of the Nd:YAG laser. As with the driver laser, iris $i 1$ partially removes unwanted beam modes, and lens $l 1(f=1 \mathrm{~m})$ focuses the beam onto the sample volume. The beam passes just over mirror $m 6$ which directs the driver beams into the test section. Note that this optical setup introduces a small angular beam misalignment with $\phi_{1}=\phi_{2}$. Mirror $m 7$ directs the scattered beam through iris $i 3$ into the receiver unit where lens L3 focuses it on pinhole (ph) with a diameter of $400 \mu \mathrm{m}$.

The signal beam is detected by a photomultiplier tube (PMT) (Hamamatsu Model OPTO-8) and recorded on a digital storage oscilloscope (DSO) (Tektronix TDS 640A) from which it is transferred over an IEEE 488 bus to a personal computer for data analysis. The transmitted part of the source (interrogation) beam is blocked as is one of the two driver beams exiting the test section. The other is detected by a photodetector (PD) (Thor Labs DET-2SI) and used to trigger the data acquisition. Each signal contains 2000 data points taken at a sampling rate of 500 Msamples/s.

\section{Procedure}

For all the experiments presented here, the test section was filled with atmospheric air seeded with $\mathrm{NO}_{2}$ at concentrations of the order of parts per million. The low level of seeding enhances the signal level by typically 2 orders of magnitude without changing the results of the measurements. Also, this seeding makes thermalization predominant over electrostriction, which can then be ignored. 
For the data analysis we used a standard personal computer (Pentium, $150 \mathrm{MHz}$ ) to determine the leastsquares fit of a theoretical signal [Eq. (17)] to an experimental trace. To keep the number of floating fitting parameters as low as possible, values for the beam diameters $\omega$ and $\sigma$, beam crossing angle $\theta$, etc. are obtained from a set of calibration measurements. A Levenberg-Marquardt scheme is used for the leastsquares fit, which is a combination of the inverse Hessian (multidimensional form of Newton's method) scheme and the method of steepest descent. ${ }^{10}$

We validated Eq. (17) by moving the translation stage with lens $l 2$ and mirror $m 6$ in the $y$ direction, thus creating a known misalignment $\bar{\eta}$. The measurement started at a value of $\bar{\eta}=-2 \mathrm{~mm}$ and a trace was recorded every $10 \mu \mathrm{m}$ until we reached $\bar{\eta}=+2$ $\mathrm{mm}$. Every trace was averaged over 64 driver laser shots to reduce the noise levels at larger misalignments.

For these measurements in which the correct beam misalignment was to be inferred from the signal shape, only the misalignment component in question and $U_{\theta}$, the thermal grating modulation depth, were adjusted during the numerical fit. The latter parameter had to be included to serve as a multiplicative factor because Eq. (17) cannot accurately give the absolute signal but rather a relative time history whose total amplitude depends additionally on the characteristics of the detector and other factors.

In a second set of measurements, we wanted to determine whether small beam misalignments affect the repeatability or accuracy of LITA measurements. This was of particular interest because it did not seem possible to detect such misalignments by the fitting procedure. Hence, at a number of fixed beam misalignments $\bar{\eta}, 500$ 64-shot averages were obtained as data for a statistical analysis. Here only the sound speeds, thermal diffusivity, and $U_{\theta}$ were adjusted during the fitting procedure. These data sets were analyzed three different ways: first, with all the misalignments (wrongly) set to zero; second, with the misalignments held fixed at their correct values; and finally with the misalignment $\bar{\eta}$ set as a fitting parameter during the data analysis.

\section{Results and Discussion}

Figure 4 shows LITA signals recorded with various misalignments together with the final results of the fitting routine for the theoretical traces. The theoretical results are shifted above the experimental signals for better comparison.

For large values of $\bar{\eta}$ [Figs. 4(a) and 4(b)], the only visible signal is due to an isolated acoustic wave passing through the source beam. We can see that the onset of the signal has a steeper slope than the tail, a result of acoustic damping. Beam diameters can be inferred accurately from these traces. The width of the hump in the signal gives a good measure of the driver beam width $\omega$. In the absence of acoustic damping, the deviation of the exact shape of the signal from a Gaussian profile gives information about the source beam diameter $\sigma$. But, because $\Gamma$ is
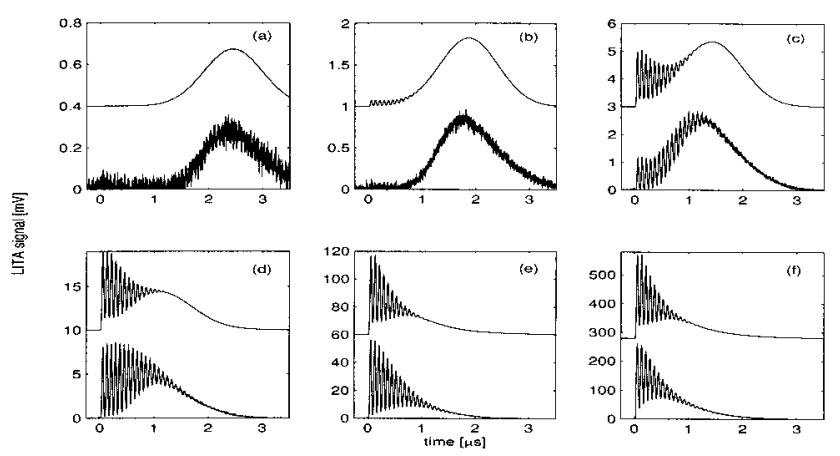

Fig. 4. Experimental and theoretical LITA signals of atmospheric air: $\sigma=700 \mu \mathrm{m}, \omega=370 \mu \mathrm{m}, \theta=1.23^{\circ}$, and (a) $\bar{\eta}=-1100 \mu \mathrm{m}$, (b) $\bar{\eta}=-900 \mu \mathrm{m}$, (c) $\bar{\eta}=-700 \mu \mathrm{m}$, (d) $\bar{\eta}=-600 \mu \mathrm{m}$, (e) $\bar{\eta}=-500$ $\mu \mathrm{m}$, and (f) $\bar{\eta}=0 \mu \mathrm{m}$. The top trace in each graph is the fitted theoretical signal, shifted for clarity.

known in these experiments, this information can still be determined by use of the least-squares fit to Eq. (17).

In Fig. 4(c) we can see the first oscillations in the signal. This indicates that at this location there is a sizable overlap of the source beam with both the thermal grating and the acoustic waves. As $\bar{\eta}$ is decreased further, the hump moves to earlier times and the oscillations become stronger. Note the subtle change in signal shape between Figs. 4(e) and 4(f) considering the large step in $\bar{\eta}$.

Figure 5 shows an experimental LITA signal together with the result of the fitting procedure. For clarity, they are plotted separately. We can see how precise Eq. (17) captures the features of a misaligned signal.

Figure 6 shows the beam misalignment inferred by the fitting routine versus the actual misalignment set by the translation stage. The dashed curves with a slope of \pm 1 correspond to the ideal case. For $\bar{\eta}>550$ $\mu \mathrm{m}$, the extracted values for $\bar{\eta}$ had the correct slope but were generally too large by approximately 200 $\mu \mathrm{m}$. For large values of $\bar{\eta}$, the larger scatter in the measurements is due to the increased noise level in

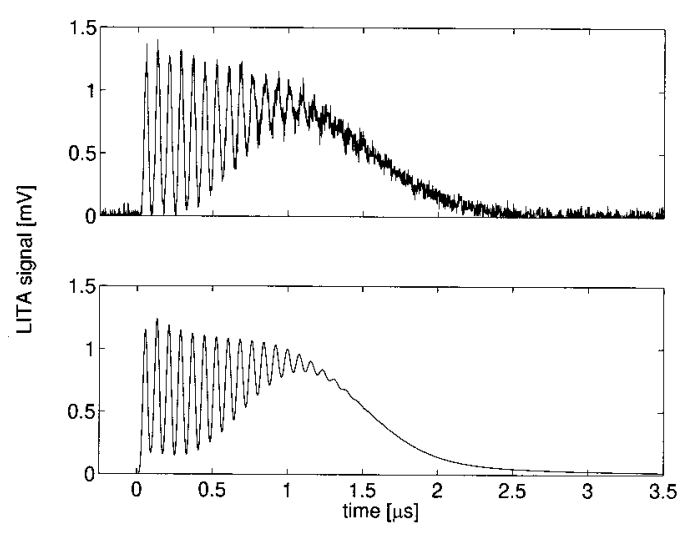

Fig. 5. Experimental signal (top) and fitted theoretical signal (bottom) for $\bar{\eta}=-1000 \mu \mathrm{m}$ with $\omega=370 \mu \mathrm{m}, \sigma=700 \mu \mathrm{m}$, and $\theta=$ $1.23^{\circ}$ in atmospheric air. 


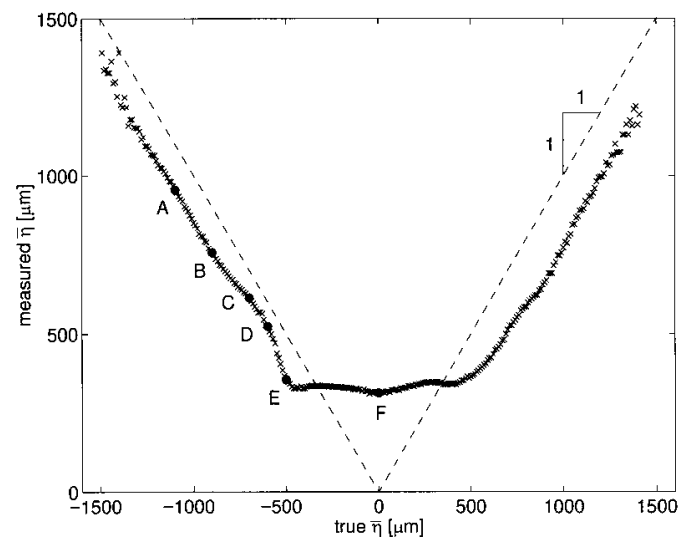

Fig. 6. Measured versus true misalignment $\bar{\eta}$. The dashed curves have a slope of \pm 1 . Traces shown in Figs. 4(a)-4(f) correspond to data points labeled A-F. The experimental conditions are the same as in Fig. 4.

the signals. A linear regression for the region $|\bar{\eta}|>$ $600 \mu \mathrm{m}$ gives slopes in these regions of $m=-0.946$ and $m=0.995$, respectively, with a coefficient of determination $r^{2}$ greater than 0.98 . The standard error for the measured $\bar{\eta}$ is $22 \mu \mathrm{m}$. The labeled data points $\mathrm{A}$ through $\mathrm{F}$ correspond to the traces shown in Figs. 4(a)-4(f).

In the center region, the detected misalignment forms a plateau at $300 \mu \mathrm{m}$. The reason for this behavior is probably that the intensity profiles of the beams in the experiments are not well represented by Gaussians. Figure 7, which shows the peak signal intensity versus $\bar{\eta}$, also provides evidence that this assumption is not satisfied. If we give the fitting routine the option of an additional fitting parameter whose effect on the signal depends strongly on the beam profiles, e.g., misalignment, while not accurately modeling the beam profiles, it is unlikely that the fitting routine will return the proper value of the parameter.

The dashed curve in Fig. 7 represents the theoretical value of the (visible) peak intensity. The inten-

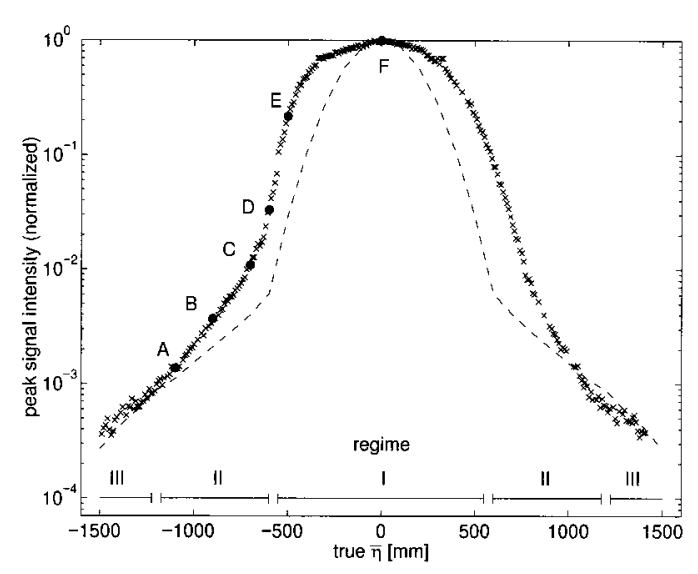

Fig. 7. Normalized peak signal intensity versus $\bar{\eta}$. Traces shown in Figs. 4(a)-4(f) correspond to data points labeled A-F. The experimental conditions are the same as in Fig. 4. sities are normalized with the intensity at $\bar{\eta}=0$. First, we consider the theoretical curve in which we can distinguish three regimes.

For $|\bar{\eta}|<575 \mu \mathrm{m}$ (regime I) we find a Gaussian behavior for the signal intensity with respect to $\bar{\eta}$. Here the peak value occurs early on in the LITA signal [Figs. 4(e) and 4(f)] because of constructive interference of the thermal grating with the acoustic waves. Both gratings have a Gaussian profile so the peak intensity scales the same way.

In regime II $(575 \mu \mathrm{m} \leq|\bar{\eta}| \leq 1200 \mu \mathrm{m})$ the peak intensity occurs at later times in the signal when an isolated acoustic wave passes the interrogation beam [Figs. 4(a) and 4(b)]. The travel time of the wave scales linearly with the misalignment. The wave's amplitude decreases exponentially with the acoustic damping rate $\Gamma$. This explains the linear behavior on a logarithmic plot for large misalignments in Fig. 7. Figures 4(c) and 4(d) mark the transition between these two regimes.

For $|\bar{\eta}|>1200 \mu \mathrm{m}$ (regime III) the theoretical signal intensity decreases again faster than in region II. This is an artifact of the limited duration of the data acquisition. In this regime, the peak of the hump visible in Figs. 4(a) and 4(b) occurs after the end of the recording time.

The measured peak intensities are higher than predicted over a range of misalignments near perfect alignment before they approach the theoretical values at larger $\bar{\eta}$. The most likely explanation is the

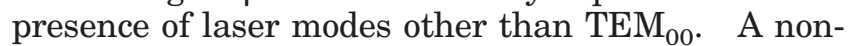
Gaussian beam profile could also be the explanation for the fact that the oscillations decay more slowly than the theory predicts for the given (fixed) beam diameters [Figs. 4(c)-4(e)].

We can see the transition from regime I to regime II in Fig. 7. The transition to III is less pronounced as at this point signals are weak. We can see the effect of noise from the large amount of scatter in the intensities in Fig. 7.

As evidenced by Fig. 6 it was not possible to extract accurate measurements of small misalignments from the signal shape. This finding prompts the question of whether small misalignments have an impact on the accuracy and uncertainty of other LITA measurements. Figures 8(a) and 8(b), respectively, show the uncertainty and the accuracy of LITA measurements of sound speed versus $\bar{\eta}$ for the three different fitting strategies.

All three fitting strategies yield almost identical results. For $|\bar{\eta}| \leq 300 \mu \mathrm{m}$ the uncertainty remains approximately constant at $0.1 \%$ as does the error of $0.2 \%$, where the measured sound speed from the data set with $\bar{\eta}=0$ has been taken as a reference value. Only for $|\bar{\eta}|>300 \mu \mathrm{m}$ do the uncertainty and error increase significantly.

Other types of misalignment that have been carried out experimentally include measurements in which only one driver beam was displaced in the $y$ direction, thus inducing a simultaneous misalignment in $\bar{\eta}$ and $\eta$, movement of both beams in opposite directions (only $\eta$ ) and measurements with misalign- 

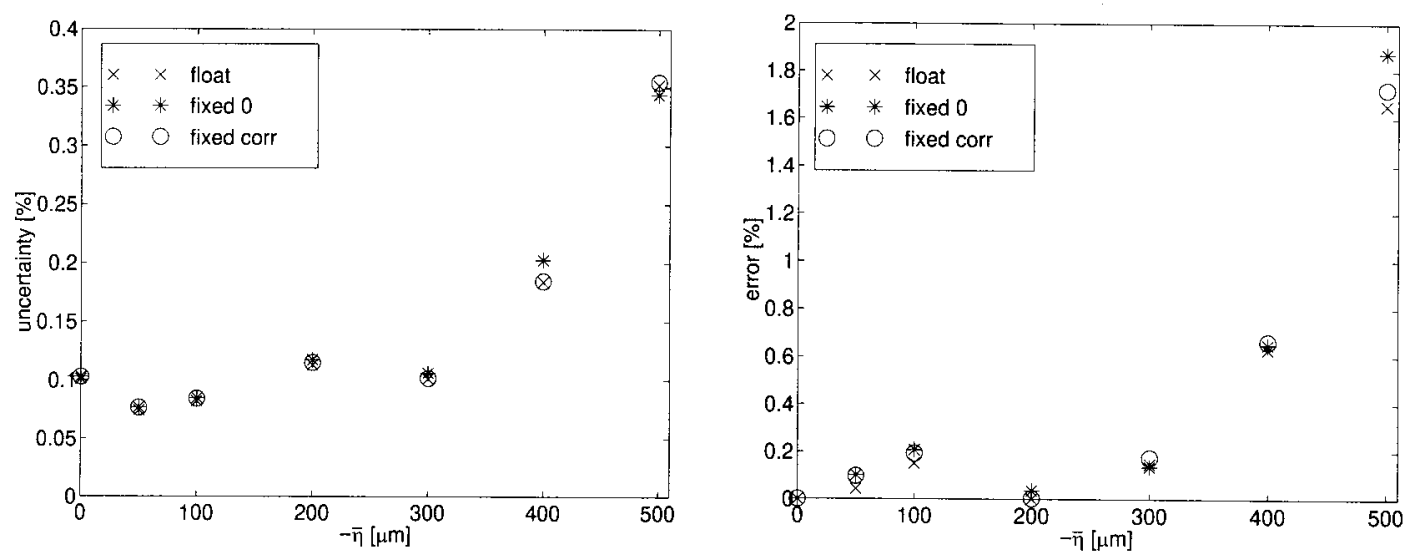

Fig. 8. Uncertainty and error of sound speed versus $\bar{\eta}$. The sound speed for $\bar{\eta}=0$ is taken as reference. Three different fitting strategies were used. In the strategy denoted by float, $\bar{\eta}$ is a floating fitting parameter; in fixed 0 , $\bar{\eta}$ is fixed at zero; and in fixed corr, $\bar{\eta}$ was held constant at the correct preset value. The experimental conditions are the same as in Fig. 4.

ments in the $z$ direction, i.e., sweeps through $\bar{\zeta}$ and $\zeta$. Misalignments in the $y$ direction produced similar results to those explained in detail above. For nonzero values of $\zeta$ or $\bar{\zeta}$, no change in signal shape was observed as Eq. (17) predicts.

When a small-diameter, $40-\mu \mathrm{m}$ pinhole was used in the receiver unit, another form of misalignment was observed that is not included in Eq. (17). Changes in signal shape did not appear to be symmetric with respect to $\bar{\eta}$. The most likely explanation for this asymmetry is that part of the signal beam was occluded by the pinhole. An illustration of this effect is shown in Fig. 9. In the upper half of the figure we can see the intensity of the overlap of the density
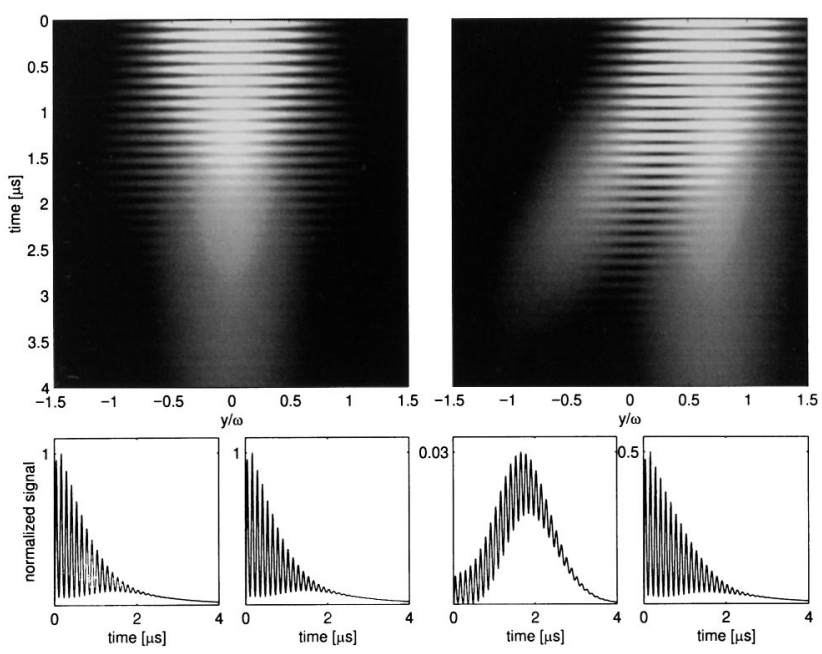

Fig. 9. Intensity of overlap of density grating with source beam from the inverse Fourier transform of Eqs. (14) for atmospheric air and at $z=0$, i.e., along the grating center line. In the upper portion, the time history as a function of $y$ position is plotted-on the left-hand side for perfect beam alignment and on the righthand side with $\bar{\eta}=\omega$. The experimental parameters are $\sigma=\omega=$ $500 \mu \mathrm{m}, \lambda_{d}=589 \mathrm{~nm}$, and $\theta=0.8^{\circ}$. White corresponds to high intensities, black to low intensities. In the lower portion, the resulting LITA signals are shown if the receiver only detects the range $y<0$ or only $y>0$. grating with the source beam in physical space along the $y$ axis as it develops over time. The bright and dark stripes correspond to the oscillations in the early stages of the signal, whereas the bright tail reflects the exponentially decaying tail of the LITA signal.

In the large detector limit we assume that the detector integrates over the entire horizontal range in Fig. 9 (and beyond). Imagine that half of the signal beam is blocked so that the receiver sees only the left or right half of the signal beam (receiver misalignment). The resulting LITA signals are given in the lower half of Fig. 9. We can see that for $\bar{\eta}=0$ this has no influence on the signal shape. In fact, we could take any region along the $y$ axis and end up with the same signal shape, meaning that this kind of misalignment cannot be detected if the optics are otherwise perfectly aligned.

However, if $\bar{\eta} \neq 0$, then the time histories at different positions $y$ are no longer self-similar, resulting in different signals depending on which part of the signal beam the detector sees.

Figure 10 provides evidence that detector misalignment produces asymmetry in beam misalignment effects. Here two traces taken at $\bar{\eta}= \pm 650 \mu \mathrm{m}$ are shown from earlier measurements with the $40-\mu \mathrm{m}$ pinhole. We can clearly see the difference in the signal shape. Compare Fig. 10 with the two traces in the lower right-hand side of Fig. 9.

To verify the origin of the asymmetry fully, we performed a series of measurements using the $40-\mu \mathrm{m}$ pinhole in which, for several values of $\bar{\eta}$, the mirror $m 7$ was translated to block different regions of the signal beam. The influence of receiver misalignment on the signal shape was demonstrated, but because this form of misalignment is not included in Eq. (17), no quantitative data can be given. Subsequently, use of a larger size pinhole eliminated the asymmetry at the cost of increased sensitivity to incoherently scattered light and luminosity.

Finally, we consider the case of angular beam misalignments and misalignments in the $x$ direction. Both can produce a rotation of the principal axes of 

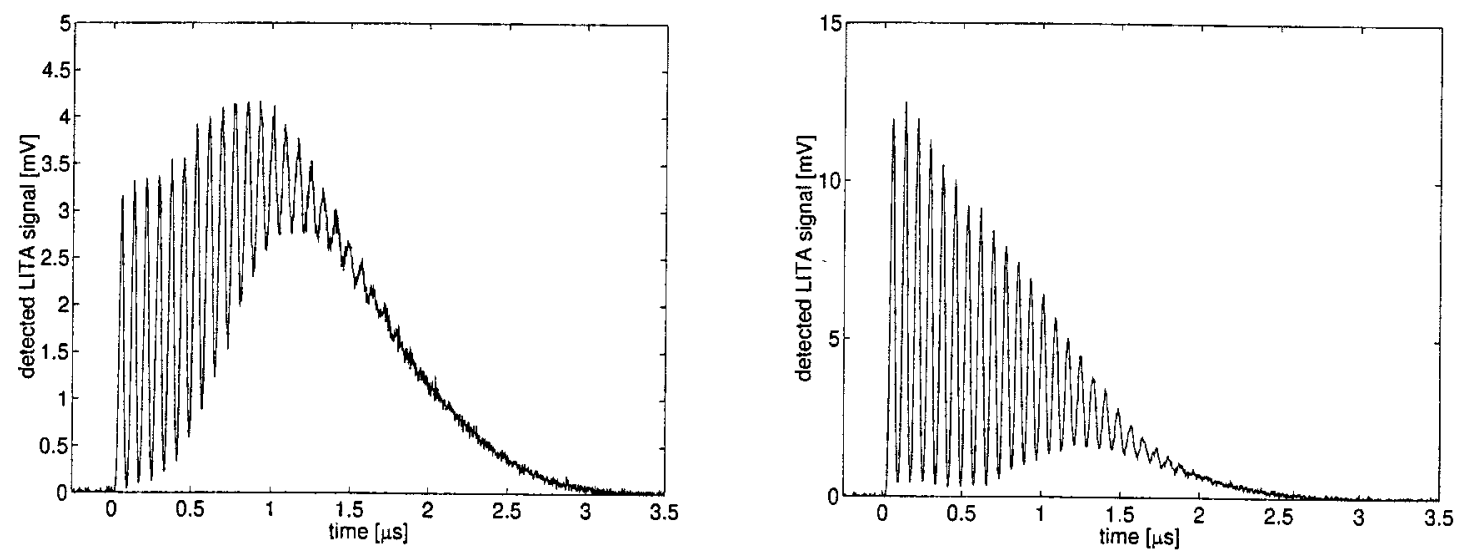

Fig. 10. Two traces from a sweep through values of $\bar{\eta}$. Left trace, $\bar{\eta}=-650 \mu \mathrm{m}$; right trace, $\bar{\eta}=+650 \mu \mathrm{m}$. A small pinhole with a diameter of only $40 \mu \mathrm{m}$ in the receiver unit blocked portions of the signal beam. Compare with theoretical results in Fig. 9.

the grating structure. As a consequence, the phasematching condition for the source beam may no longer be satisfied, resulting in a weaker signal beam that may even be scattered into a different direction than anticipated. This alone, however, does not change the shape of the signal as long as the entire signal beam falls on the detector.

\section{Conclusions}

An analytical expression for the magnitude and time history of LITA signals from finite Gaussian beams in the presence of fluid velocities and the most common forms of beam misalignment has been derived. In experiments, some deviations from this expression were observed that were due to non-Gaussian laser beam profiles. In regions where this assumption was satisfied, however, the experiments showed good agreement with the theory.

It has been demonstrated how beam misalignments can be detected quantitatively from only the shape of the signal. Small misalignments that could not be measured accurately were shown not to influence the accuracy or repeatability of sound speed measurements, even when the data analysis incorrectly assumed that no misalignments were present during the measurement.

Beam misalignment information provided by this technique can be used in the initial optical setup and to correct in real time for slow-occurring misalignments, e.g., because of thermal expansion. The time scale at which misalignments can be corrected is limited by the repetition rate of the driver laser so that a real-time alignment is not possible for turbulent flows where refractive-index gradients may introduce random, nonstationary beam misalignments. In these situations it will be necessary to allow for beam misalignments in the data analysis.

Controlled beam misalignments may be advanta- geous for optimizing the accuracy of measurements of a particular parameter. For example, beam misalignments can improve the accuracy of homodyne LITA velocimetry.

The authors acknowledge Thomas H. Sobota of Advanced Projects Research, Incorporated, for providing the motivation for understanding and characterizing the effects of beam misalignments and fluid velocity on the LITA signal. This research was supported by Advanced Projects Research, Incorporated, and the U.S. Air Force Research Laboratory at Edwards Air Force Base.

\section{References}

1. T. J. Butenhoff, "Measurement of the thermal-diffusivity and speed of sound of hydrothermal solutions via the laser-induced grating technique," Int. J. Thermophys. 16 (1), 1-9 (1995).

2. B. Hemmerling and D. N. Kozlov, "Generation and temporally resolved detection of laser-induced gratings by a single, pulsed Nd:YAG laser," Appl. Opt. 38, 1001-1007 (1999).

3. M. S. Brown and W. L. Roberts, "Single-point thermometry in high-pressure, sooting, premixed combustion environments," J. Propul. Power 15, 119-127 (1999).

4. D. J. W. Walker, R. B. Williams, and P. Ewart, "Thermal grating velocimetry," Opt. Lett. 23, 1316-1318 (1998).

5. E. B. Cummings, I. A. Leyva, and H. G. Hornung, "Laserinduced thermal acoustics (LITA) signals from finite beams," Appl. Opt. 34, 3290-3302 (1995).

6. H. J. Eichler, P. Günther, and D. W. Phol, Laser-Induced Dynamic Gratings (Springer-Verlag, New York, 1986).

7. D. B. Brayton, "Small particle signal characteristics of a dualscatter laser velocimeter," Appl. Opt. 13, 2346-2351 (1974).

8. A. E. Siegman, "Bragg diffraction of a Gaussian beam by a crossed-Gaussian volume grating," J. Opt. Soc. Am. 67, 545550 (1977).

9. B. J. Berne and R. Pecora, Dynamic Light Scattering (Wiley, New York, 1976).

10. W. H. Press, Numerical Recipes in C: The Art of Scientific Computing (Cambridge U. Press, New York, 1988). 